\title{
Removal of transient disturbances from oscillating measurements using nearest neighbors imputation
}

\author{
Inês M. Cecílio ${ }^{\mathrm{a}, *}$, James R. Ottewill ${ }^{\mathrm{b}}$, Harald Fretheim ${ }^{\mathrm{c}}$, Nina F. Thornhill ${ }^{\mathrm{a}}$ \\ ${ }^{a}$ Centre for Process System Engineering, Department of Chemical Engineering, Imperial College \\ London, London SW7 2AZ, UK \\ ${ }^{b} A B B$ Corporate Research Center, ul. Starowiślna 13a, 31-038 Kraków, Poland \\ ${ }^{c}$ ABB Oil, Gas and Petrochemicals, Ole Deviks Vei 10, 0666 Oslo, Norway
}

\begin{abstract}
Transient disturbances in process measurements compromise the accuracy of some methods for plant-wide oscillation analysis. This paper presents a method to remove such transients while maintaining the dynamic features of the original measurement. The method is based on a nearest neighbors imputation technique. It replaces the removed transient with an estimate which is based on the time series of the whole measurement. The method is demonstrated on experimental and industrial case studies. The results demonstrate the efficacy of the method and recommended parameters. Furthermore, inconsistency indices are proposed which facilitate the automation of the method.
\end{abstract}

Keywords: Transients, oscillations, plantwide, disturbances, nearest neighbors, imputation, nonlinear time series analysis.

\section{Introduction}

Detection and diagnosis of plant-wide oscillations has been an active topic of research in the process and control loop monitoring communities. Oscillatory disturbances are dynamic disturbances that persist over a time horizon. In the time domain, measurements affected by oscillatory disturbances show repeating patterns. Often, the repeating patterns have non-sinusoidal shapes and may repeat intermittently, as exemplified in figures later in this paper (Figure 4, Figure 15). As a result, in the frequency domain these disturbances can be characterized by single or multiple spectral peaks, or broad-band features [1].

\footnotetext{
${ }^{*}$ Corresponding author

Email address: i.cecilio09@imperial.ac.uk (Inês M. Cecílio )
} 


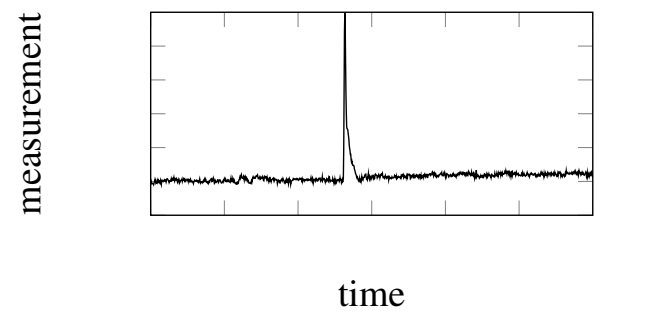

(a) Response to impulse-like disturbance

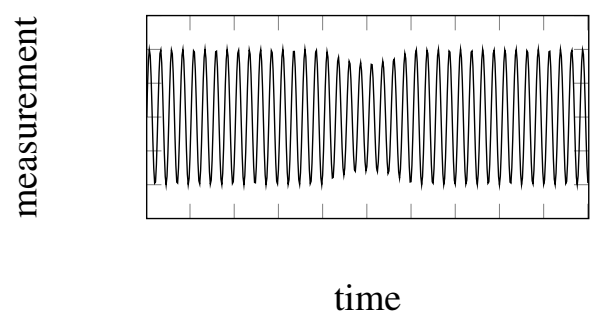

(c) Voltage sag

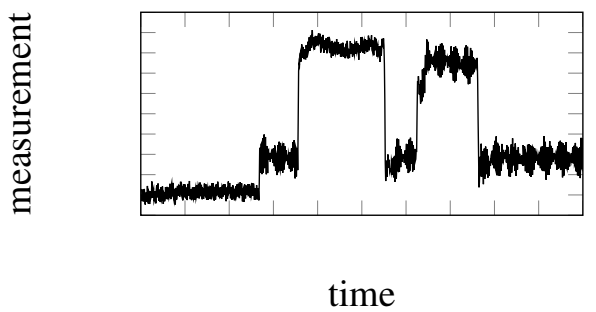

(b) Responses to step changes
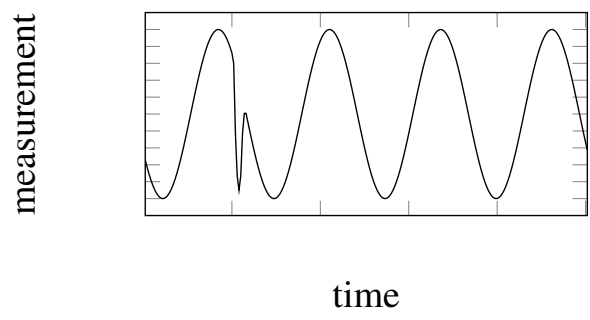

(d) Voltage transient

Figure 1: Examples of transient disturbances

As discussed in review papers [1,2], the methods developed to analyse oscillating disturbances are data-driven, and most of them extract properties from the time series of a process measurement or from the relations between time series of different measurements. An example is the non-linearity of the time series of a measurement. The more non-linear the time series is, the closer the measurement should be to the root cause of a nonlinear oscillating disturbance [3, 4]. Outside the analysis of plant-wide oscillations, Qin [5] has reviewed data-driven methods of process monitoring which use statistical data-based models, and Venkatasubramanian et al. $[6,7,8]$ have surveyed the use of data-based, analytical, and qualitative models.

It has been reported in the literature that the accuracy of oscillation analysis methods can be affected by the presence of unrelated transient disturbances [9]. A transient disturbance is a particular class of deviations in the trend of a system variable, which is defined by being short-lasting and seldom repeated within the time horizon of analysis [10]. Figure 1 shows examples of transient disturbances. These include spikes (Figure 1a and Figure 1d) and sags (Figure 1c), after which the system variable often returns to its underlying trend, as well as the responses of the system to step changes (Figure 1b), after which the time series follows a different trend. 
Transient disturbances modify time and frequency properties of the time series on which some oscillation analysis methods rely. According to the valve stiction review book by Jelali and Scali [9], examples of methods affected by outliers, sharp abrupt changes, and step changes include the methods of bicoherence [3] and surrogate analysis [4].

It would thus be interesting to automatically detect transient disturbances in oscillating measurements, and to remove the transients without significantly altering the underlying dynamics of the original measurements. As discussed below, important contributions have already been made to the detection of transient disturbances, but the removal of transients from oscillating measurements remains a challenge.

The detection of transient disturbances in process measurements has been addressed with Qualitative Trend Analysis (QTA), data-based models, wavelet decomposition, and nearest neighbors methods. Related work outside process and control loop monitoring includes the detection of edges in images [11], whereby edges are defined as step changes which can be masked by noise. QTA detects the transient disturbance by comparing its time trend with basic shapes [12]. As a result, QTA requires the creation of a complete database with all possible shapes of a transient disturbance. The traditional methods that use data-based models are known as statistical process monitoring, and assume that the time-domain magnitude of the transient disturbance is higher than the normal trend of the measurement [13]. As a result, statistical process monitoring methods would not recognize the transient disturbance in a measurement with oscillatory or cyclical dynamics if the transient had lower magnitude than the oscillations. Wavelet decomposition [14] is able to detect transient disturbances which map to wavelet coefficients in the lower scales, provided that the non-transient parts of the measurement do not produce other high-amplitude coefficients at those same scales. However, Cecílio et al. [15] showed an example of an oscillating measurement in which this condition was not met. The nearest neighbors method proposed by Cecílio et al. [10, 15] is more generic than the previous approaches because it does not require the transient disturbance to have a specific shape, a higher magnitude or distinct wavelet coefficients. Instead, the method detects any rare and short-lasting deviation of a measurement from its previous and subsequent trend.

The removal of disturbance-related values from the time series of process measurements is often known as fault reconstruction [5]. The reconstructed values are estimates of what the time series would have been had the disturbance not been present. Existing methods require a statistical data-based model. This model reveals the relationships between the time series process measurements under normal operating conditions. Therefore, with such a model available, the reconstructed time series can be recalculated as linear combinations of all measurements avail- 
able $[16,17,18]$. The data-based model most commonly derives from Principal Component Analysis (PCA) or Partial Least Squares (PLS) analysis of the matrix of process measurements $[19,5]$. Although in principle valid for any type of disturbance, the use of data-based models for removal of disturbances is limited to systems and types of operation for which accurate models have been built. Furthermore, this approach requires the knowledge of which measurements show the disturbance, and the existence of sensor redundancy [18].

Filtering in the frequency domain is a fundamental tool to isolate periodic oscillations in a measurement. This type of analysis determines the amplitude spectrum of the measurement, for example through Fast Fourier Transform, removes the range of undesired frequencies, and reconstructs the filtered time series. This approach, however, is not appropriate for removing transient disturbances because the frequency content of a transient is spread across the whole frequency spectrum. Also, this approach modifies the whole measurement, both in the removed frequencies and in the artifacts introduced by the filter, such as spectral leakage. These effects are not desirable when the objective is to carry out an oscillation analysis after the reconstruction.

The fields of image and speech processing have also devoted attention to decomposing a signal into oscillating components of different frequency contents [20]. However, as discussed, transient disturbances may not have a defined frequency range. Conversely, a more recent contribution [20] proposes a method to distinguish oscillating from transient components by the extent to which they are sustained, whereby sustained is defined as some number of consecutive oscillating cycles. However, as discussed at the start, oscillating disturbances in real process systems may repeat intermittently. Hence it could be ambiguous to judge whether the repeating patterns are sustained. Other works decompose the image or speech signal into linear combinations of orthonormal bases, formulating the decomposition as an optimization problem [21].

The method proposed in this paper removes transients from time series with oscillating disturbances. The method does not require the existence of models, and the time series is only modified locally, in the segment affected by the transient. The only requirement for the method is that the underlying trend of the time series has repetitive patterns, as exemplified in figures later in the paper (Figure 4,Figure 3 and Figure 15).

The proposed method is based on a nearest neighbors imputation technique. This technique handles the measurement of a process variable as a time series, and replaces the removed transient disturbance with a segment of time series which agrees with the underlying trend of the original measurement. In other words, the replacing segment is an estimate of what the measurement would have been had the transient not been present. 
The paper is organized as follows. Section 2 presents the background on nonlinear deterministic systems, and on how nearest neighbors techniques can take advantage of those systems for the purpose of imputation. Section 3 presents the algorithm. The section starts by introducing the case study on which the algorithm is demonstrated. This section also discusses criteria to evaluate the adequacy of the replacing segment. Using those criteria, section 4 analyzes the sensitivity of the results to the parameters of the algorithm. The analyses lead to clear recommendations for the values of the parameters. Finally, section 5 tests the method and parameters on a case study from an industrial gas processing plant. The paper closes with comments and a summary.

\section{Background}

This section introduces the concept of non-linear deterministic systems, and explains how nearest neighbors techniques can determine unknown segments in time series generated from those systems.

\subsection{Non-linear deterministic systems}

A deterministic system is defined by the fact that its present state unambiguously determines its future state. Mathematically, such systems are described by ordinary differential equations, or their equivalent discrete-time maps,

$$
\mathbf{x}_{i+1}=\mathbf{F}\left(\mathbf{x}_{i}\right), \quad i \in \mathbb{Z} .
$$

The state of a system is observed through a measurement $X$ of that system. The state at instant $i$ is defined by the ordered sequence of $M$ samples of $X$

$$
\mathbf{x}_{i}=\left[\begin{array}{llll}
x_{i} & x_{i+1} & \cdots & x_{i+M-1}
\end{array}\right] .
$$

The number $M$ of samples which defines the state is such that the condition (1) of determinism is verified.

The group of all possible states of a deterministic system defines its state space. Figure 2 shows the state space for a system which generates sinusoidal time series. In such a system, a dimension $M=2$ is enough to observe its determinism. This is visible in Figure 2 by the well defined structure in the two-dimensional plot.

In non-linear systems, the mapping $\mathbf{F}$ is non-linear. A linear system depends on a linear combination of past values, while a non-linear system has terms such as $x_{i-1}^{2}$ or $x_{i} \times x_{i-1}$. Kantz and Schreiber [22] present a comprehensive explanation of non-linear systems, and methods to analyse their time series. 


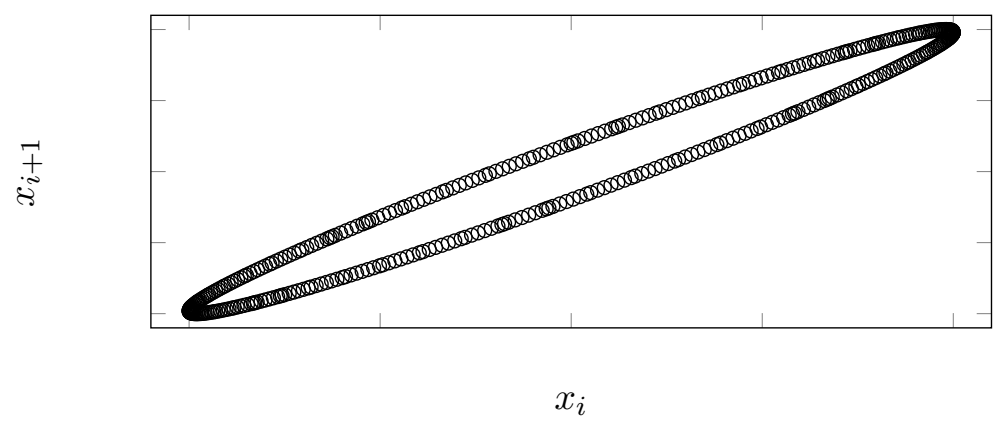

Figure 2: State space of dimension $M=2$ for a sinusoidal time series.

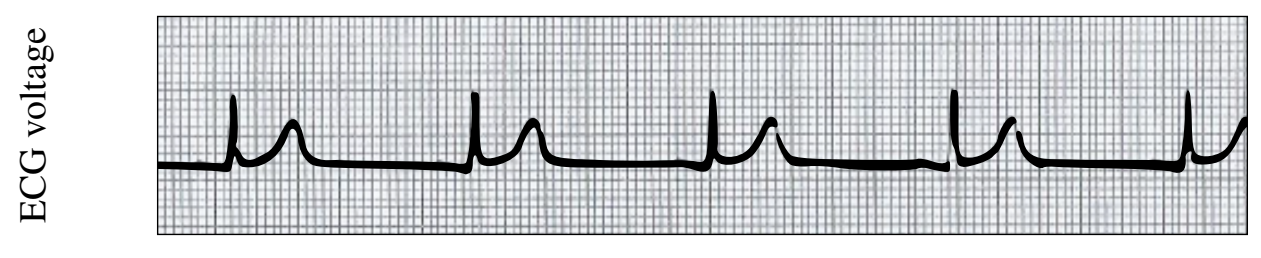

time

Figure 3: Example of non-linear oscillatory time series: the cycles are clearly non-sinusoidal.

\subsection{Predictability in non-linear deterministic systems}

If the mapping $\mathbf{F}$ were known, then future states $\mathbf{x}_{i+1}$ of the system could be predicted from past states $\mathbf{x}_{i}$. Even if this is not the case, predictability can be guaranteed if the mapping $\mathbf{F}$ is assumed to be continuous. If this is true, then any two $\mathbf{x}_{i}$ states which are close in the state space will lead to future $\mathbf{x}_{i+1}$ states which are also close. If one of these future states were unknown, the other would be a good predictor. The best predictor $\mathbf{x}_{i+1}$ is that with the most similar past state $\mathbf{x}_{i}$.

Therefore, in the absence of the mapping $\mathbf{F}$, prediction of future state is possible given a sufficiently long history of the system where states similar to the present can be found. Systems with oscillatory behavior normally show several repetitions of the same state, and thus are appropriate for this type of prediction. A simple example is a system resulting in purely periodic time series. Once one full cycle is observed, the rest of the time series is known to be a repetition of that same cycle.

Most real oscillatory time series are non-linear and hence not purely periodic. Non-linear oscillatory time series show repeated states with non-sinusoidal shapes and which may appear intermittently. Two examples are shown in Figure 3 and Figure 4. 


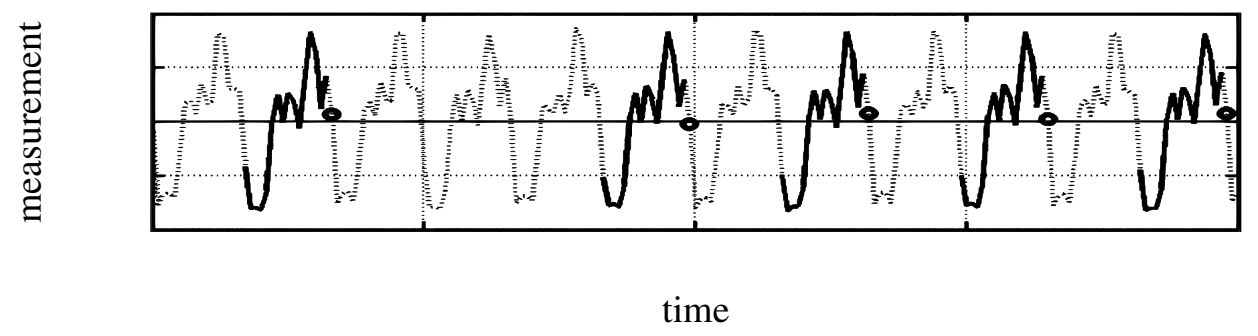

Figure 4: A non-linear deterministic time series with five nearest neighbor cycles followed by five similar points (adapted from Thornhill [4]).

Figure 4 further illustrates the determinism of the non-linear oscillatory time series plotted. The time series is not purely periodic because the cycles are not all equal. Nonetheless, five cycles with similar sequences of samples can be identified (highlighted in thick lines). Due to determinism, these cycles are followed by five similar samples (marked with circles).

\subsection{Nearest neighbor imputation}

Real systems may not be purely deterministic. Even if they are, the measurements through which they are observed are often affected by random inputs, such as instrument noise. In such cases, using a single past state to predict the unknown state is not necessarily the most accurate predition.

In order to be robust to outliers and uncertainty, the method of nearest neighbor imputation [22] searches for the $k$ states which are the most similar to the past of the unknown state. The $k$ most similar states are known as the $k$ nearest neighbors. The Euclidean distance is a common metric to assess similarity between segments of time series. Cecílio et al. [10] discussed other similarity metrics and their adequacy for finding nearest neighbors. The $k$ states after the nearest neighbors are then averaged to form the predictor of the unknown future state. Kantz and Schreiber [22] place no constraints on the number $k$ of elements of the nearest neighborhood. The choice of $k$ is discussed in detail in section 4.2.3.

Nearest neighbor imputation has been used to test the determinism [23] and the non-linearity of a time series [4], to replace missing values in survey data [24], to determine the precedence relationship between two measurements [25], and to determine the time lag between two measurements [26]. In particular, references $[4,25]$ and [26] reported applications in process systems. The measurements in these cases originated from processes under the influence of non-linear oscillatory disturbances, such as non-linear hydrodynamic instabilities and limit cycles generated by control valves with excessive static friction. This paper focuses on mea- 


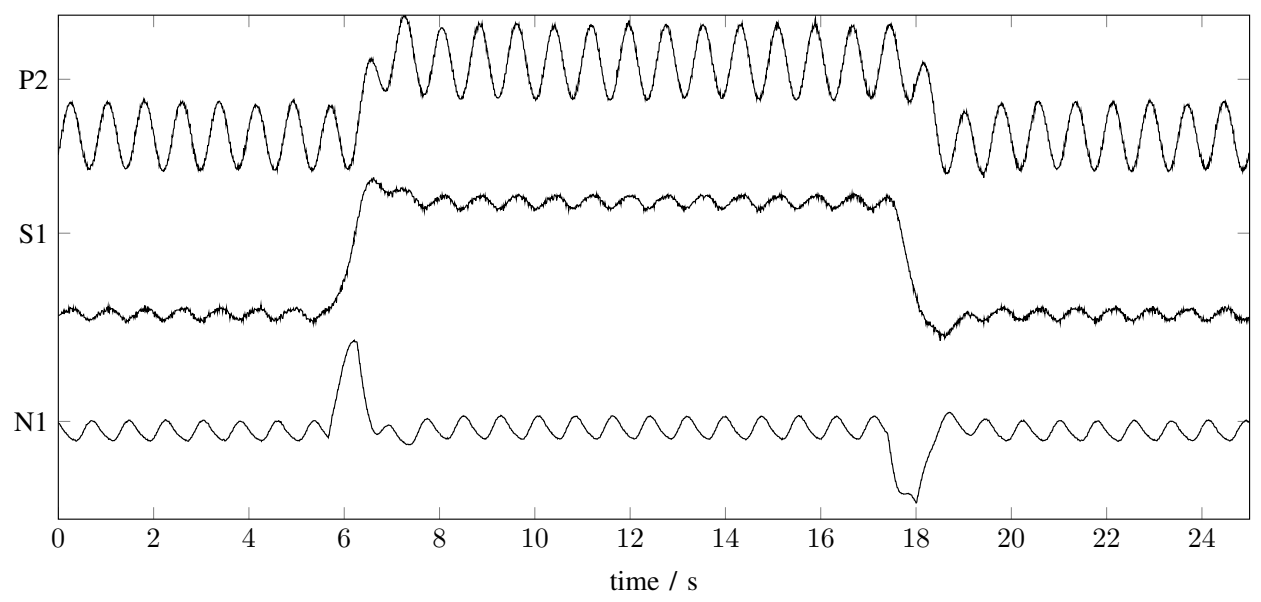

Figure 5: Development case study: time series of measurements from a gas compression rig.

surements whose underlying dynamic trends are oscillatory, hence the motivation to use nearest neighbor imputation.

\section{Method}

This section explains the method proposed to remove transient disturbances from the time series of an oscillating measurement. A development case study is first presented in order to illustrate the explanation. The end of the section discusses criteria to evaluate the adequacy of the segment which replaces the removed transient.

\subsection{Development case study}

Figure 5 shows the time trends of three measurements obtained from experiments with a gas compression rig. The rig is located at ABB Corporate Research Center, Kraków, Poland. Figure 6 shows a schematic of this rig in order to situate the measurement tags.

The measurements are affected by an oscillatory disturbance which is due to compressor surge. Furthermore, the three measurements clearly show two transient disturbances, around $6 \mathrm{~s}$ and $18 \mathrm{~s}$. The objective is to replace the segments affected by the transients with estimates that agree with the underlying time series.

\subsection{Algorithm}

The method handles a measurement as a time series $X$. Therefore, the transient disturbance can be associated with the segment of the time series that starts at time 


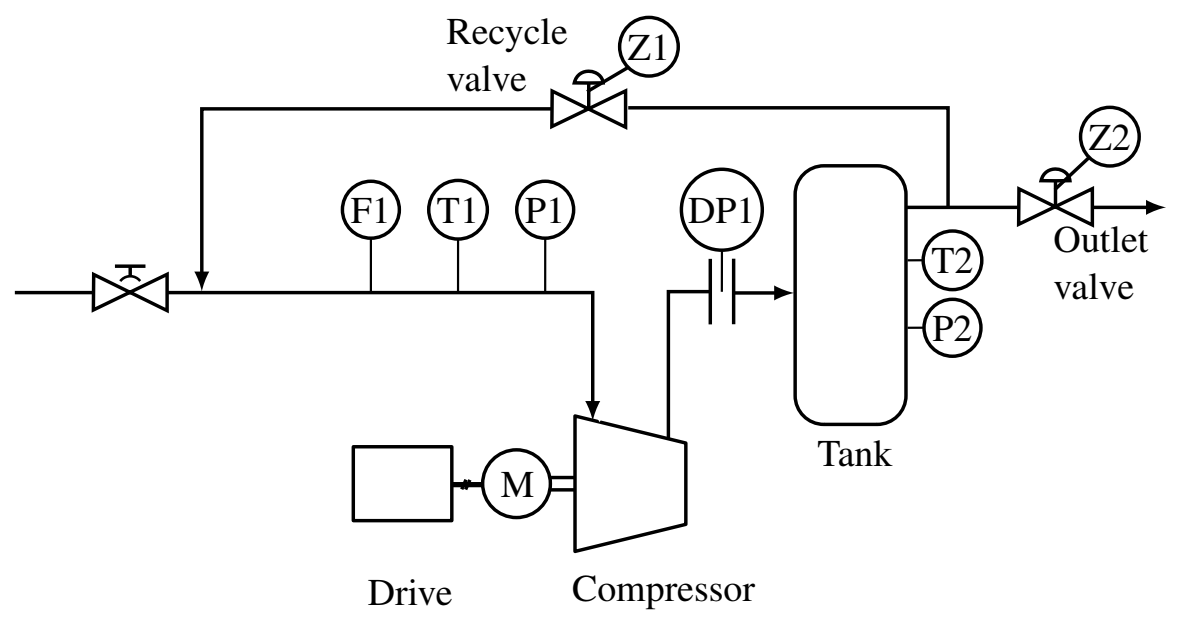

Figure 6: Simplified schematic of the gas compression experimental rig.

instant $t_{i}$ and ends at time instant $t_{f}$, that is $\left[x_{t_{i}} \cdots x_{t_{f}}\right]$. Automated methods to locate the transient disturbances are found in the literature and were discussed in the Introduction. Figure 7 shows a close-up of measurement S1. The light grey segment corresponds to the transient disturbance.

The first step of the method is to remove the segment $\left[x_{t_{i}} \cdots x_{t_{f}}\right]$ from time series $X$. The objective is to replace segment $\left[x_{t_{i}} \cdots x_{t_{f}}\right]$ by a different segment, $\left[\begin{array}{lll}\hat{x}_{t_{i}} & \cdots & \hat{x}_{t_{f}}\end{array}\right]$, which agrees with the overall dynamics of time series $X$. The replacing segment $\left[\hat{x}_{t_{i}} \cdots \hat{x}_{t_{f}}\right]$ will be computed in the next subsections using the property of predictability of deterministic systems (section 2 ).

\subsubsection{States and embedding vectors}

A state is represented by $M$ samples. This is the dimension for which the system is deterministic. States are approximated by embedded vectors which span those $M$ samples. An embedded vector $\mathbf{x}_{i}$ is formed by $m$ samples, $\tau$ instants apart, that is,

$$
\mathbf{x}_{i}=\left[\begin{array}{llll}
x_{i} & x_{i+\tau} & \cdots & x_{i+(m-1) \tau}
\end{array}\right] .
$$

The dimension $M$ is related to $m$ and $\tau$ according to $M=(m-1) \tau+1$. The reason to consider $\tau$ greater than one is to minimize the computational effort of the algorithm. This is discussed in section 4 .

The embedded vectors generated from time series $X$ can be arranged in the rows of an embedding matrix, as shown in equation (4). 


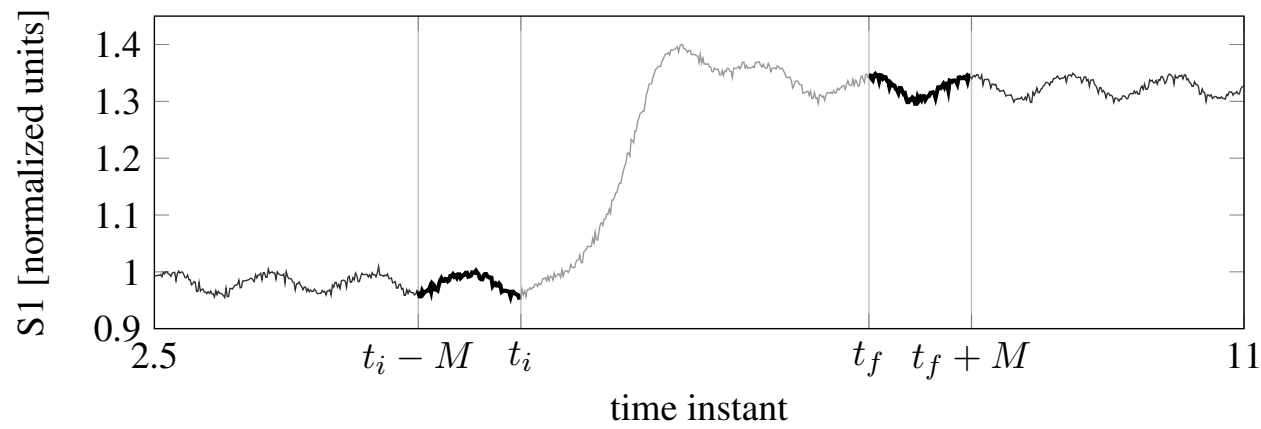

Figure 7: Close-up of measurement S1 highlighting the transient (grey line) and the pre- and posttransient references (black thick lines).

$\mathbf{X}=\left[\begin{array}{c}\mathbf{x}_{1} \\ \mathbf{x}_{2} \\ \vdots \\ \mathbf{x}_{N_{E}}\end{array}\right]=\left[\begin{array}{cccc}x_{1} & x_{1+\tau} & \cdots & x_{1+(m-1) \tau} \\ x_{1+\delta} & x_{1+\delta+\tau} & \cdots & x_{1+\delta+(m-1) \tau} \\ \vdots & \vdots & \vdots & \\ x_{1+\left(N_{E}-1\right) \delta} & x_{1+\left(N_{E}-1\right) \delta+\tau} & \cdots & x_{1+\left(N_{E}-1\right) \delta+(m-1) \tau}\end{array}\right]$

Each embedded vector in the matrix lags the previous by $\delta$ samples. The number $N_{E}$ of embedded vectors depends on parameters $m, \tau$, and $\delta$, as well as on the total number of samples $n$. The use of $\delta$ greater than one corresponds to omitting states of the time series. As with $\tau$, the reason may be to minimize the computational effort of the algorithm.

Before further processing, each embedded vector in the matrix is centered around its own mean. This is done to enable the recognition of embedded vectors that have the same dynamic behaviour, even if they are centered around a different mean value. Examples of such embedded vectors are those before $t_{i}$ and after $t_{f}$ in Figure 7.

\subsubsection{Pre- and post-transient references}

The embedded vectors $\mathbf{x}_{t_{i}-M}$ and $\mathbf{x}_{t_{f}}$ are those immediately before and immediately after the removed segment $\left[x_{t_{i}} \cdots x_{t_{f}}\right]$. For the development case study, Figure 7 highlights $\mathbf{x}_{t_{i}-M}$ and $\mathbf{x}_{t_{f}}$ with thick black lines. Embedded vectors $\mathbf{x}_{t_{i}-M}$ and $\mathbf{x}_{t_{f}}$ are denoted as pre-transient reference and post-transient reference. Once the transient is removed, they will be used to predict the empty samples between $t_{i}$ and $t_{f}$.

If the system generating time series $X$ is purely deterministic, then the prediction based on the pre-transient reference $\mathbf{x}_{t_{i}-M}$ will be as correct as the prediction 
based on the post-transient reference $\mathbf{x}_{t_{f}}$. The reason is that in purely deterministic systems, the mapping $\mathbf{x}_{i+1}=\mathbf{F}\left(\mathbf{x}_{i}\right)$ is continuous. However, it is unlikely that real systems are purely deterministic. Therefore, the samples $\left[\hat{x}_{t_{i}} \cdots \hat{x}_{t_{f}}\right]$ predicted by the pre-transient reference and by the post-transient reference may be different. The prediction which fits better to the time series is not known in advance, hence the method computes the two predictions $\left[\hat{x}_{t_{i}} \cdots \hat{x}_{t_{f}}\right]$ in parallel, and assesses the adequacy of each at the end. The predictions based on the pre-transient reference and on the post-transient reference have similar algorithms. Therefore, the following description only highlights the necessary differences.

\subsubsection{Similarity}

For both references $\mathbf{x}_{t_{i}-M}$ and $\mathbf{x}_{t_{f}}$, the similarity between the reference and all other embedded vectors is assessed with the Euclidean distance metric. For the pre-transient reference $\mathbf{x}_{t_{i}-M}$ and a generic embedded vector $\mathbf{x}_{j}$, the Euclidean distance is defined as

$$
d\left(\mathbf{x}_{t_{i}-M}, \mathbf{x}_{j}\right)=\sqrt{\sum_{i=1}^{m}\left(x_{t_{i}-M+(i-1)}-x_{j+(i-1)}\right)^{2}} .
$$

With the embedded vectors arranged in the embedding matrix, the implementation of this similarity assessment is straightforward.

\subsubsection{Nearest neighbors of the pre- and post-transient references}

Nearest neighbors of a reference denote the embedded vectors which are the most similar to the reference, as indicated by the smallest distance measures. The

$k$ nearest neighbors of the pre-transient reference $\mathbf{x}_{t_{i}-M}$ are represented by $\mathbf{x}_{t_{j}}^{t_{i}-M}$, with $j=1 \cdots k$. The $k$ nearest neighbors of the post-transient reference $\mathbf{x}_{t_{f}}$ are represented by $\mathbf{x}_{t_{j}}^{t_{f}}$, with $j=1 \cdots k$.

Figure 8 shows the complete measurement $\mathrm{S} 1$, and highlights the $k=2$ nearest neighbors of the pre-transient reference $\mathbf{x}_{t_{i}-M}$ for the first transient. The pretransient reference is enclosed by a dashed rectangle, while its $k=2$ nearest neighbors are highlighted with thick black lines.

Some embedded vectors are excluded from being considered near neighbors of the pre- and post-transient references. The next subsection will clarify the reasons for this. Table 1 indicates that the excluded embedded vectors are at one of the extreme ends of the time series, as well as next to and including a transient disturbance.

Figure 8 highlights with grey lines the embedded vectors which were excluded from the neighborhood assessment for the pre-transient reference of the first transient. 


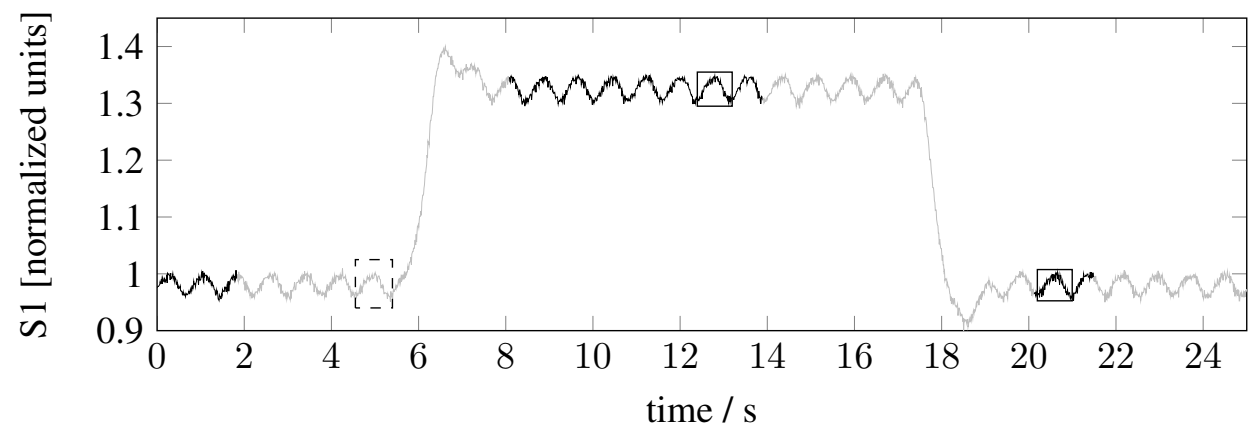

Figure 8: Measurement S1 highlighting the $k=2$ nearest neighbors (continuous rectangles) of the pre-transient reference of the first transient (dashed rectangle). The grey lines indicate the embedded vectors excluded from the neighborhood assessment.

Table 1: Embedded vectors excluded from the neighborhood assessment of the pre- and post-transient references. Excluded embedded vectors are at one of the extreme ends of the time series $X$, and next to and including a transient disturbance. $T=t_{f}-t_{i}$, and $n$ is the total number of samples of $X$.

\begin{tabular}{lccc}
\hline Location of embedded vectors & Pre-transient & Post-transient \\
\hline $\begin{array}{l}\text { Extreme end } \\
\text { of time series }\end{array}$ & start & - & $\mathbf{x}_{1} \cdots \mathbf{x}_{1+T}$ \\
\hline Next to and including & after & $\mathbf{x}_{n-M-T} \cdots \mathbf{x}_{n-M}$ & - \\
transient $\left[x_{t_{i}} \cdots x_{t_{f}}\right]$ & before & $\mathbf{x}_{t_{i}-M-T} \cdots \mathbf{x}_{t_{f}-M}$ & $\mathbf{x}_{t_{i}} \cdots \mathbf{x}_{t_{f}+T}$ \\
\hline
\end{tabular}

\subsubsection{Replacing segment}

For each nearest neighbor $\mathbf{x}_{t_{j}}^{t_{i}-M}=\left[\begin{array}{lll}x_{t_{j}}^{t_{i}-M} & \cdots & x_{t_{j}+M-1}^{t_{i}-M}\end{array}\right]$ of the pre-transient reference $\mathbf{x}_{t_{i}-M}$, its subsequent segment $\left[\begin{array}{lll}x_{t_{j}+M}^{t_{i}-M} & \cdots & x_{t_{j}+M+T}^{t_{i}-M}\end{array}\right]$ is considered, where $T=t_{f}-t_{i}$.

Each segment $\left[\begin{array}{lll}x_{t_{j}+M}^{t_{i}-M} & \cdots & x_{t_{j}+M+T}^{t_{i}-M}\end{array}\right]$ is mean centered, and the mean-centered segments are denoted as $\left[\begin{array}{lll}x_{0, t_{j}+M}^{t_{i}-M} & \cdots & x_{0, t_{j}+M+T}^{t_{i}-M}\end{array}\right]$. The $k$ mean-centered segments are averaged according to

$$
\left[\begin{array}{ccc}
\frac{1}{k} \sum_{j=1}^{k} x_{0, t_{j}+M}^{t_{i}-M} & \cdots & \frac{1}{k} \sum_{j=1}^{k} x_{0, t_{j}+M+T}^{t_{i}-M}
\end{array}\right] .
$$

The mean-centering step is required because of time series with non-flat trends. The reference case study is an example. As shown in Figure 8, the segments 
$\left[\begin{array}{lll}x_{t_{j}+M}^{t_{i}-M} & \cdots & x_{t_{j}+M+T}^{t_{i}-M}\end{array}\right]$ after the two nearest neighbors follow the same trend, but are centered around a different mean value.

Equation (6) results in the segment $\left[\hat{x}_{t_{i}} \cdots \hat{x}_{t_{f}}\right]$, which replaces the empty samples between $t_{i}$ and $t_{f}$. Segment $\left[\begin{array}{lll}\hat{x}_{t_{i}} & \cdots & \hat{x}_{t_{f}}\end{array}\right]$ should agree with the overall dynamics of time series $X$.

Segment $\left[\begin{array}{lll}\hat{x}_{t_{i}} & \cdots & \hat{x}_{t_{f}}\end{array}\right]$ can also derive from the post-transient reference $\mathbf{x}_{t_{f}}$. In this case, the previous segment $\left[\begin{array}{lll}x_{t_{j}-T}^{t_{f}} & \cdots & x_{t_{j}-1}^{t_{f}}\end{array}\right]$ of each nearest neighbor $\mathbf{x}_{t_{j}}^{t_{f}}=\left[\begin{array}{lll}x_{t_{j}}^{t_{f}} & \cdots & x_{t_{j}+M-1}^{t_{f}}\end{array}\right]$ is considered. The mean-centering and averaging steps are applied on the $k$ segments $\left[\begin{array}{lll}x_{t_{j}-T}^{t_{f}} & \cdots & x_{t_{j}-1}^{t_{f}}\end{array}\right]$.

The previous subsection indicated that some embedded vectors are excluded from being considered near neighbors of the pre- and post-transient references. The reason is that the replacing segment $\left[\hat{x}_{t_{i}} \cdots \hat{x}_{t_{f}}\right]$ is defined by the $T$-sample segments after the nearest neighbor, in the case of the pre-transient reference, or before the nearest neighbors in the case of the post-transient reference. Therefore, embedded vectors at one of the ends of the time series, as well as next to and including a transient disturbance need to be excluded.

\subsubsection{Concatenation of segments and reconstructed time series $Y$}

A reconstructed time series $Y$ without transients is generated by concatenating the replacing segments $\left[\hat{x}_{t_{i}} \cdots \hat{x}_{t_{f}}\right]$ and the transient-free segments of the original time series.

As with the generation of the replacing segment, the concatenation procedure needs to be robust to time series with non-flat trends. The concern in the steps described below is to avoid introducing artificial discontinuities. Discontinuities would arise when adjoining segments centered around a different mean value, such as the segments before $t_{i}$ and after $t_{f}$ in Figure 7.

Figure 9 illustrates the concatenation procedure based on the pre-transient references $\mathbf{x}_{t_{i}-M}$, and the list below describes the steps.

1. The first segment of the reconstructed time series $Y$ is the first transient-free segment of the original time series $X$, that is,

$$
\left[\begin{array}{llll}
y_{1} & y_{2} & \cdots & y_{t_{i}-1}
\end{array}\right]=\left[\begin{array}{llll}
x_{1} & x_{2} & \cdots & x_{t_{i}-1}
\end{array}\right]
$$

2. The numerical values of the replacing segment $\left[\hat{x}_{t_{i}} \cdots \hat{x}_{t_{f}}\right]$ are shifted to match the point $y_{t_{i}-1}$ according to

$$
\left[\begin{array}{lll}
y_{t_{i}} & \cdots & y_{t_{f}}
\end{array}\right]=\left[\begin{array}{lll}
\hat{x}_{t_{i}} & \cdots & \hat{x}_{t_{f}}
\end{array}\right]-\left(\hat{x}_{t_{i}}-\left(y_{t_{i}-1}+\Delta y_{t_{i}-1}\right)\right)
$$




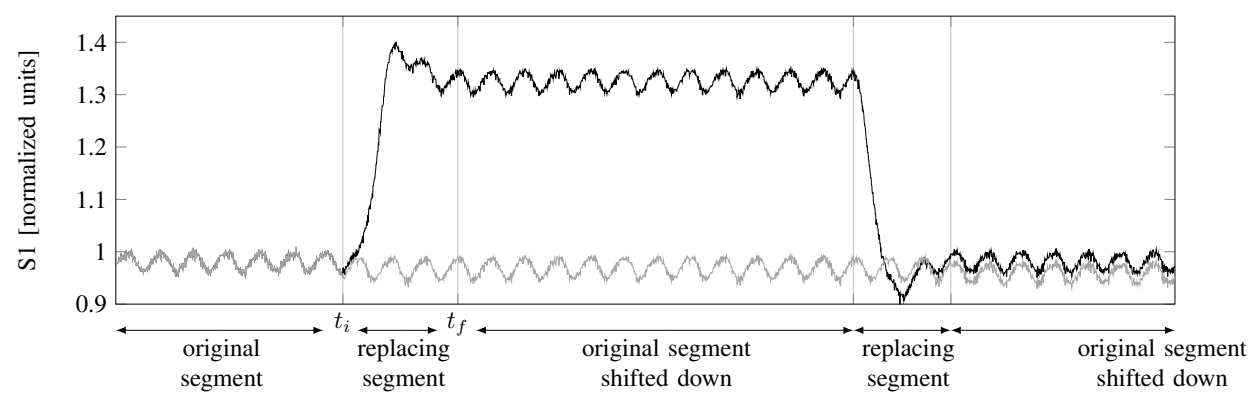

Figure 9: Generation of the reconstructed time series (grey line) by concatenating the replacing segments, and the transient-free segments of the original time series (black line).

where $\Delta y_{t_{i}-1}$ is the average of the differences at the end of each nearest neighbor $\mathbf{x}_{t_{j}}^{t_{i}-M}$, that is,

$$
\Delta y_{t_{i}-1}=\frac{1}{k} \sum_{j=1}^{k}\left(x_{t_{j}+M}^{t_{i}-M}-x_{t_{j}+M-1}^{t_{i}-M}\right)
$$

The purpose of shifting is to avoid discontinuities between the two adjoining segments. The difference $\Delta y_{t_{i}-1}$ estimates what $\Delta x_{t_{i}-1}=x_{t_{i}}-x_{t_{i}-1}$ would have been if the transient disturbance had not occurred.

3. The next transient-free segment of the original time series is the one which starts at $x_{t_{f}+1}$ and ends before the next transient disturbance. The numerical values of this transient-free segment are shifted to match the point $y_{t_{f}}$ according to

$$
\left[\begin{array}{lll}
y_{t_{f}+1} & \cdots & y_{t_{i, 2}}
\end{array}\right]=\left[\begin{array}{lll}
x_{t_{f}+1} & \cdots & x_{t_{i, 2}}
\end{array}\right]-\left(x_{t_{f}+1}-\left(y_{t_{f}}+\Delta y_{t_{f}}\right)\right)
$$

where the time instant $t_{i, 2}$ refers to the start of the second transient disturbance. The value $\Delta y_{t_{f}}$ is the average of the differences at the start of each nearest neighbor $\mathbf{x}_{t_{j}}^{t_{f}}$. Therefore, $\Delta y_{t_{f}}$ approximates what the difference $\Delta x_{t_{f}}=x_{t_{f}+1}-x_{t_{f}}$ would have been if not for the transient.

These shifting steps are repeated for the following replacing segments and transient-free segments of the original time series $X$.

The concatenation procedure based on the post-transient reference $\mathbf{x}_{t_{f}}$ is done in the opposite direction, that is, from the end of the reconstructed time series $Y$ to its start.

Both Figure 10a and Figure 10b show the original time series $X$ of the measurements in the development case study in black lines. Superimposed on these 


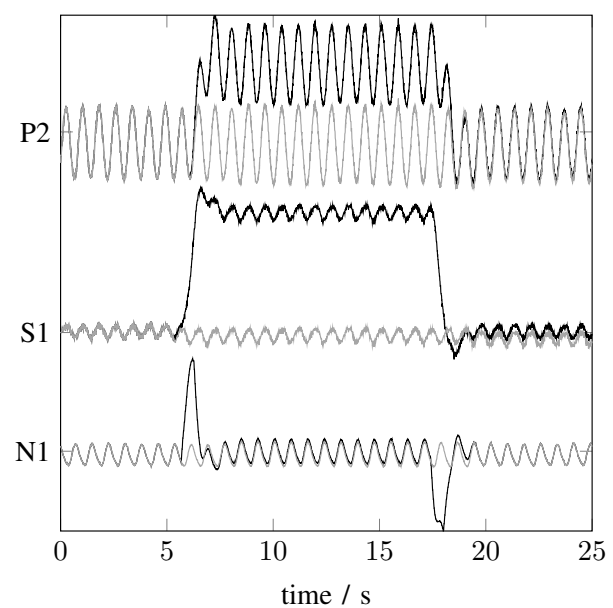

(a) Based on the pre-transient references.

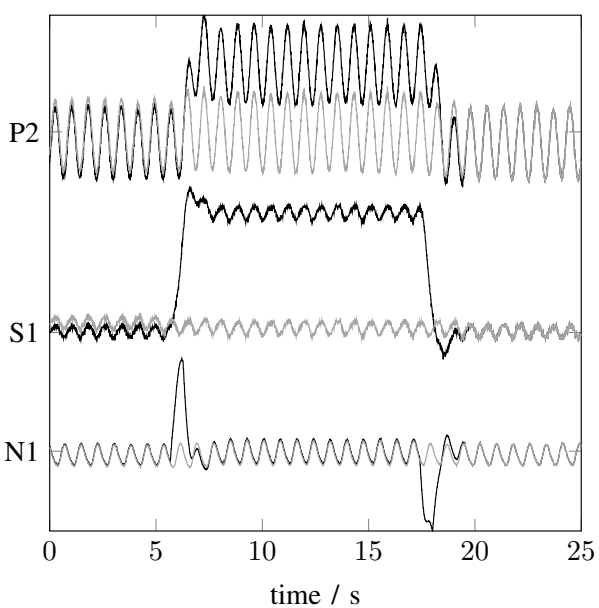

(b) Based on the post-transient references.

Figure 10: Reconstructed time series (grey lines) after removal of transients, superimposed on the original time series (black lines).

and in grey lines, Figure 10a shows the reconstructed time series based on the pretransient references $\mathbf{x}_{t_{i}-M}$, and Figure $10 \mathrm{~b}$ shows the reconstructed time series based on the post-transient reference $\mathbf{x}_{t_{f}}$.

\subsection{Adequacy of the replacing segment}

The concern with the removal of transient disturbances is that the replacing segment should agree with the underlying transient-free time series. To assess possible mismatches, this section proposes the two following indices:

- Index $D_{k}$ measures how similar the reference embedded vector is to its $k$ nearest neighbors.

- Index $s_{k}$ measures how similar the replacing segment is to the $k$ meancentered segments which are averaged into it (equation 6).

Indices $D_{k}$ and $s_{k}$ should be minimized because they assess inconsistencies in the prediction of the replacing segments. In particular, if the system generating the time series is purely deterministic, and the choice of dimension $M$ is correct, then $D_{k}$ and $s_{k}$ should be zero. In this case, the replacing segment $\left[\hat{x}_{t_{i}} \cdots \hat{x}_{t_{f}}\right]$ will exactly match the underlying time series.

For both the pre- and post-transient references, index $D_{k}$ is the average of the squared distances between the reference embedded vector and its $k$ nearest 
neighbors. This criterion is normalized by the variance of the reference embedded vector. Equation (11) describes the computation of $D_{k}$ from the pre-transient reference $\mathbf{x}_{t_{i}-M}=\left[\begin{array}{lll}x_{t_{i}-M} & \cdots & x_{t_{i}-1}\end{array}\right]$ and its nearest neighbors $\mathbf{x}_{t_{j}}^{t_{i}-M}=$ $\left[\begin{array}{lll}x_{t_{j}}^{t_{i}-M} & \cdots & x_{t_{j}+M-1}^{t_{i}-M}\end{array}\right]$.

$$
D_{k}=\frac{1}{k} \sum_{j=1}^{k}\left(\frac{\sum_{i=1}^{M}\left(x_{t_{i}-M+(i-1)}-x_{t_{j}+(i-1)}^{t_{i}-M}\right)^{2}}{\sum_{i=1}^{M}\left(x_{t_{i}-M+(i-1)}-\bar{x}_{t_{i}-M}\right)^{2}}\right)
$$

The variance of the reference embedded vector, in the denominator of equation (11), is equivalent to the square of the distance between the reference embedded vector and its mean, respectively $\mathbf{x}_{t_{i}-M}$ and $\bar{x}_{t_{i}-M}$ in the case of the pre-transient reference. As a result, the normalization proposed compares the similarity of the $k$ nearest neighbors to the similarity of the mean of the reference embedded vector.

Index $s_{k}$ is the average of the squared distances between the replacing segment $\left[\hat{x}_{t_{i}} \cdots \hat{x}_{t_{f}}\right]$ and the $k$ mean-centered segments which are averaged into it. This criterion is normalized by the variance of the replacing segment. Equation (12) describes the computation of $s_{k}$ based on the pre-transient reference $\mathbf{x}_{t_{i}-M}$. In this case, the $k$ mean-centered segments are denoted by $\left[\begin{array}{lll}x_{0, t_{j}+M}^{t_{i}-M} & \cdots & x_{0, t_{j}+M+T}^{t_{i}-M}\end{array}\right]$.

$$
s_{k}=\frac{1}{k} \sum_{j=1}^{k}\left(\frac{\sum_{i=1}^{T+1}\left(\hat{x}_{t_{i}+(i-1)}-x_{0, t_{j}+M+(i-1)}^{t_{i}-M}\right)^{2}}{\sum_{i=1}^{T+1}\left(\hat{x}_{t_{i}+(i-1)}-\overline{\hat{x}}\right)^{2}}\right),
$$

where $T=t_{f}-t_{i}$. The variance of the replacing segment, in the denominator of equation (12), is equivalent to the square of the distance between the replacing segment and its mean $\overline{\hat{x}}$. As a result, the normalization proposed compares the similarity of the $k$ mean-centered segments to the similarity of the mean of the replacing segment.

For the development case study, Table 2 indicates the values of $D_{k}$ and $s_{k}$ for the replacing segments based on the pre- and post-transient references. The values are averaged over the two transient disturbances.

Indices $D_{k}$ and $s_{k}$ are able to distinguish between the adequacy of the replacing segments which were calculated based on the pre-transient reference and on the post-transient reference. For practical applications, the reconstructed time series $Y$ of a measurement is the one that derives from the replacing segment with lower indices. 
Table 2: Inconsistency indices $D_{k}$ and $s_{k}$ based on the pre- and post-transient references for the development case study.

\begin{tabular}{lccccc}
\hline \multirow{2}{*}{ Tag } & \multicolumn{2}{c}{$D_{k}$} & & \multicolumn{2}{c}{$s_{k}$} \\
\cline { 2 - 3 } \cline { 5 - 6 } & Pre-transient & Post-transient & & Pre-transient & Post-transient \\
\hline P2 & 0.0067 & 0.0050 & & 0.0017 & 0.0007 \\
S1 & 0.1491 & 0.1225 & & 0.0029 & 0.0032 \\
N1 & 0.0049 & 0.0065 & & 0.0011 & 0.0011 \\
\hline
\end{tabular}

Figure 10 suggests that the reconstructed time series (grey lines) are equally adequate whether based on the pre- or post-transient references. For measurements $\mathrm{S} 1$ and N1, indices $D_{k}$ and $s_{k}$ in table 2 agree with this observation. In fact, the values of indices $D_{k}$ and $s_{k}$ are not consistently lower for either the pre-transient reference or the post-transient reference. On the other hand, for measurement P2 the values of indices $D_{k}$ and $s_{k}$ are both lower based on the post-transient references. This result indicates that, for measurement $\mathrm{P} 2$, the replacing segments based on the post-transient references are the ones which best agree with the underlying dynamics of the original time series.

\section{Parameter settings and sensitivity}

The removal of transient disturbances involves the following parameters:

- embedding dimension $m$,

- embedding granularity $\tau$,

- embedding step $\delta$, and

- number of nearest neighbors $k$.

The objective of this section is to arrive at guidelines for optimal parameter values.

As further explained below, parameters $m, \tau$, and $\delta$ are related to the original sampling rate of the time series as well as the dynamics of the system. Therefore, section 4.2 finds the optimal parameter values as a function of those characteristics of the system and time series. In a practical application of the method, users can thus set the optimal values from their knowledge of the sampling rate and time constants of the system. 
On the other hand, the optimal parameter $k$ depends on the individual time series, namely on the existence of outliers. Therefore, section 4.2 proposes and shows the workflow to arrive at the the optimal value of $k$ in the course of a practical application.

Before the parameter optimization, section 4.1 discusses how each parameter influences the removal task.

\subsection{Role of each parameter}

\subsubsection{Embedding granularity $\tau$ and embedding step $\delta$}

Parameters $\tau$ and $\delta$ intervene in the generation of the embedded vectors (equations (3) and (4)). In this step of the method, the parameters have an analogous effect to downsampling because $\tau>1$ discard samples from the embedded vectors and $\delta>1$ discards embedded vectors from the embedding matrix. Specifically, setting $\tau=\delta$ is equivalent to downsampling the time series by a factor of $\tau$.

Smaller values of $\tau$ improve the similarity assessment between embedded vectors because, with more samples, outliers to the trends have a smaller influence. With smaller values of $\delta$, the true nearest neighbors of the reference embedded vector should be present in the embedding matrix.

On the other hand, the algorithm is $\mathcal{O}(1 / \tau \delta)$ and thus greater values of $\tau$ and $\delta$ reduce the computational time, without having to downsample the time series. The reason to use $\tau$ and $\delta$ instead of downsampling is to retain all the samples of the original time series in the reconstructed time series. This is important because the reconstructed time series will be used in other analyses.

As this discussion elucidates, the optimal values of $\tau$ and $\delta$ depend on the relation between the main time constant of the system and the original sampling rate of the time series.

\subsubsection{Embedding dimension $m$}

As with $\tau$ and $\delta$, parameter $m$ intervenes in the generation of the embedded vectors (equations (3) and (4)). For a given $\tau$, the embedding dimension $m$ determines the span of an embedded vector as $(m-1) \tau+1$ samples. An embedded vector should span the same number of samples as a state of the system in order to exploit the determinism of the system. The state of the system, which can be seen as the repeating pattern, depends on the main time constant of the system. Therefore, the optimal value of $m$ depends on that time constant and the chosen $\tau$.

\subsubsection{Number of nearest neighbors $k$}

Parameter $k$ determines the number of nearest neighbors of the reference embedded vector and, by extension, the number of mean-centered segments which are averaged into the replacing segment $\left[\hat{x}_{t_{i}} \cdots \hat{x}_{t_{f}}\right]$. The replacing segment is an 
estimate of the missing section of the time series. The best estimate derives from the number $k$ of mean-centered segments which agree the most. Real systems are affected by outliers and uncertainty, and therefore the optimal value of $k$ depends on the individual time series being treated for transient removal.

\subsection{Recommendations for parameters and analysis of sensitivity}

This section arrives at guidelines for setting optimal parameter values. This is done by studying the behaviour of the algorithm with varying parameter values. The recommendations for parameters $\tau, \delta$, and $m$ are generic for any system. This is done by referring these parameters to $T_{P}$, the number of samples in one cycle of the oscillatory time series. The optimal value of parameter $k$ is specific for each time series, hence a generic workflow to find this value is proposed.

The analysis is carried on the development case study, as follows. Different parameter values are used in order to generate the reconstructed time series $Y$ for each time series. This is done based on the pre-transient reference and the posttransient reference of each transient disturbance. As defined in section 3.2, reference means the embedded vector immediately before (pre-transient reference) or after (post-transient reference) the removed segment $\left[x_{t_{i}} \cdots x_{t_{f}}\right]$. The adequacy of the replacing segments is evaluated based on the indices $D_{k}$ and $s_{k}$ introduced in section 3.3. These indices measure inconsistencies in the estimation of the replacing segment, and thus should be minimized.

\subsubsection{Embedding granularity $\tau$ and embedding step $\delta$}

Setting $\tau=\delta$ is equivalent to downsampling the time series by a factor of $\tau$. The optimal downsampling factor will depend on the original sampling rate. To make this optimization valid for any original sampling rate and frequency of oscillation, the embedding granularity is analysed as $T_{P} / \tau$. This ratio is equivalent to the number of samples in one cycle if the time series were actually downsampled by a factor of $\tau$.

Figure 11 shows the influence of $T_{P} / \tau$ on the indices $D_{k}$ (panel 11a) and $s_{k}$ (panel 11b). In this case study, $T_{P}$ is approximately 800 samples. Each plot in a panel shows the optimization results for each variable of the development case study. Each plot combines the results based on the pre-transient reference (black line with round marker) and the post-transient reference (grey line with cross marker).

In general, all plots show lower indices $D_{k}$ and $s_{k}$ at higher $T_{P} / \tau$, indicating that $\tau$ and $\delta$ should be equal to one. This is expected, because increasing $\tau$ and $\delta$ means discarding more information.

However, the computational time of the algorithm increases with $T_{P} / \tau$, as shown in Figure 12. Thus, if the computational time is prohibitive, it is important to note 
that above $T_{P} / \tau=40$ the improvement in performance is moderate. Specifically, the reduction in $D_{k}$ and $s_{k}$ is in general below $20 \%$.

\subsubsection{Embedding dimension $m$}

The analysis of the embedding dimension $m$ serves to indicate the optimal duration of a state in the problem of nearest neighbour imputation. The duration of a state is given by $(m-1) \tau \Delta t$. As a result, the value of parameter $m$ depends on the chosen $\tau$ and on the original sampling rate $\Delta t$. With regards to $\tau$, this optimization uses $\tau=1$, which is the optimal value. If higher values of $\tau$ are needed, then the value of $m$ must be varied accordingly. To make this optimization valid for any original sampling rate and frequency of oscillation, the embedding dimension is analysed as $m / T_{P}$.

Figure 13 shows the influence of $m$ on the indices $D_{k}$ (panel 13a) and $s_{k}$ (panel 13b). Each plot in a panel shows the optimization results for each variable of the development case study. Each plot combines the results based on the pre-transient reference (black line with round marker) and the post-transient reference (grey line with cross marker).

In general, all figures show a steep decrease in the indices $D_{k}$ and $s_{k}$ when $m$ increases until $m=T_{P}$. Above $m=T_{P}$, index $D_{k}$ increases, while $s_{k}$ stays approximately at the same level. These results suggest that, for $\tau=1, m$ should be set equal to the number of samples in one cycle, $T_{P}$. Similar conclusions are reported elsewhere [4]. 


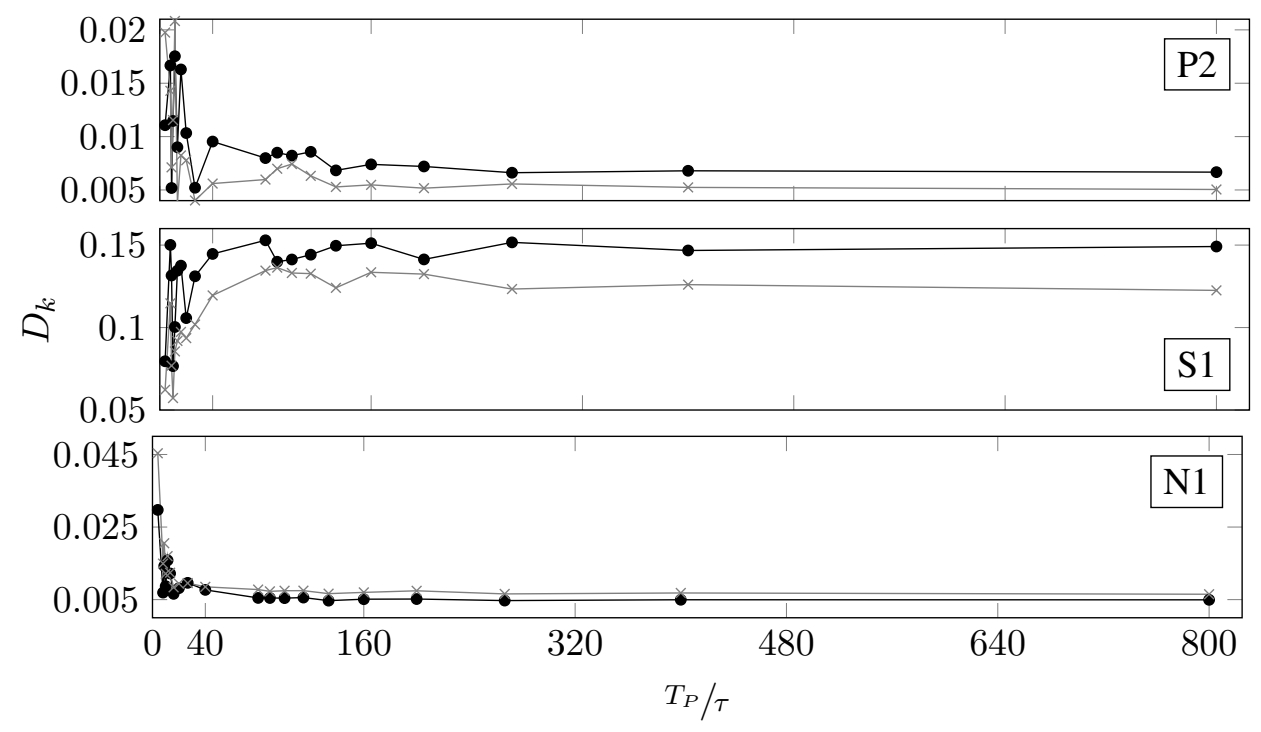

(a) Performance measure $D_{k}$.

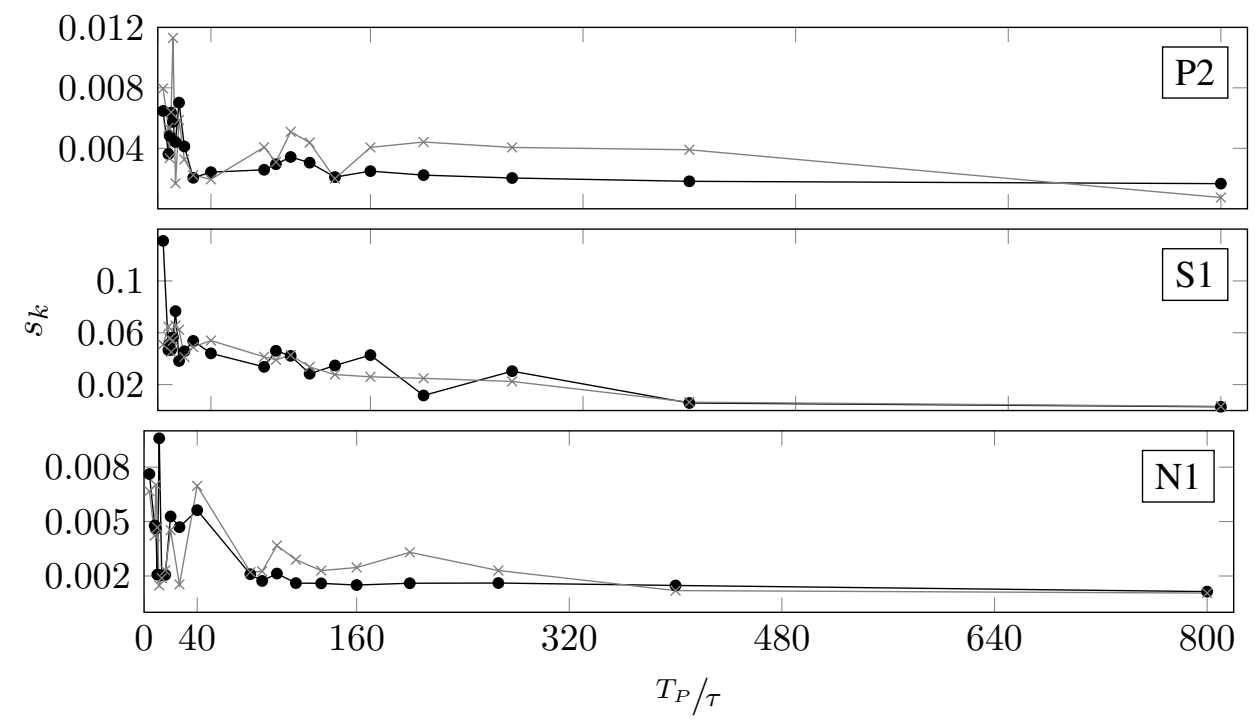

(b) Performance measure $s_{k}$.

Figure 11: Performance of the removal method as a function of $\tau$ and $\delta$, when $\tau=\delta$. Parameters fixed in the analyses were $m=800$ and $k=2$. The number of samples per cycle is $T_{P}=800$. Results are shown for each variable, and for the estimations based on the pre-transient reference (black line with round marker) and the post-transient reference (grey line with cross marker). 


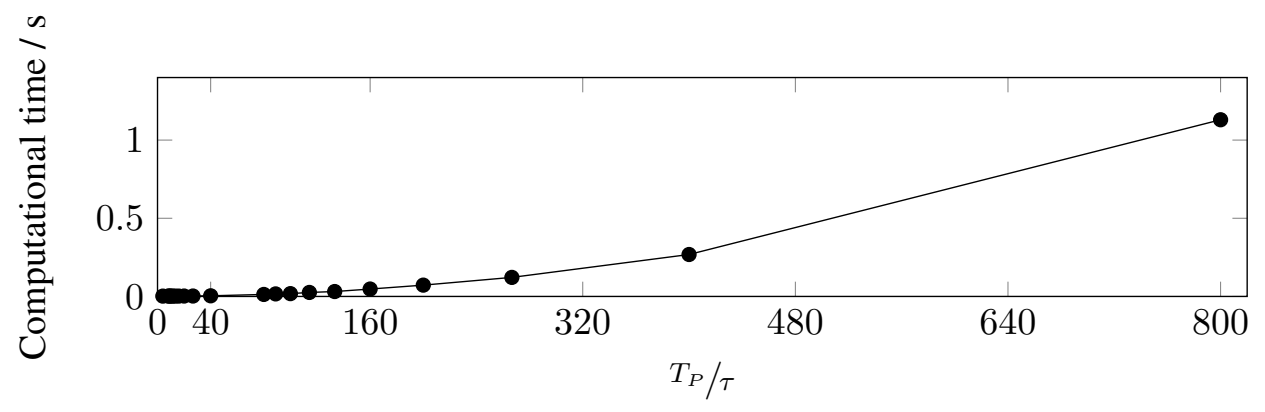

Figure 12: Computational time as a function of $T_{P} / \tau$. The computational time is an average over the three variables, and includes the removal of the two transients. 


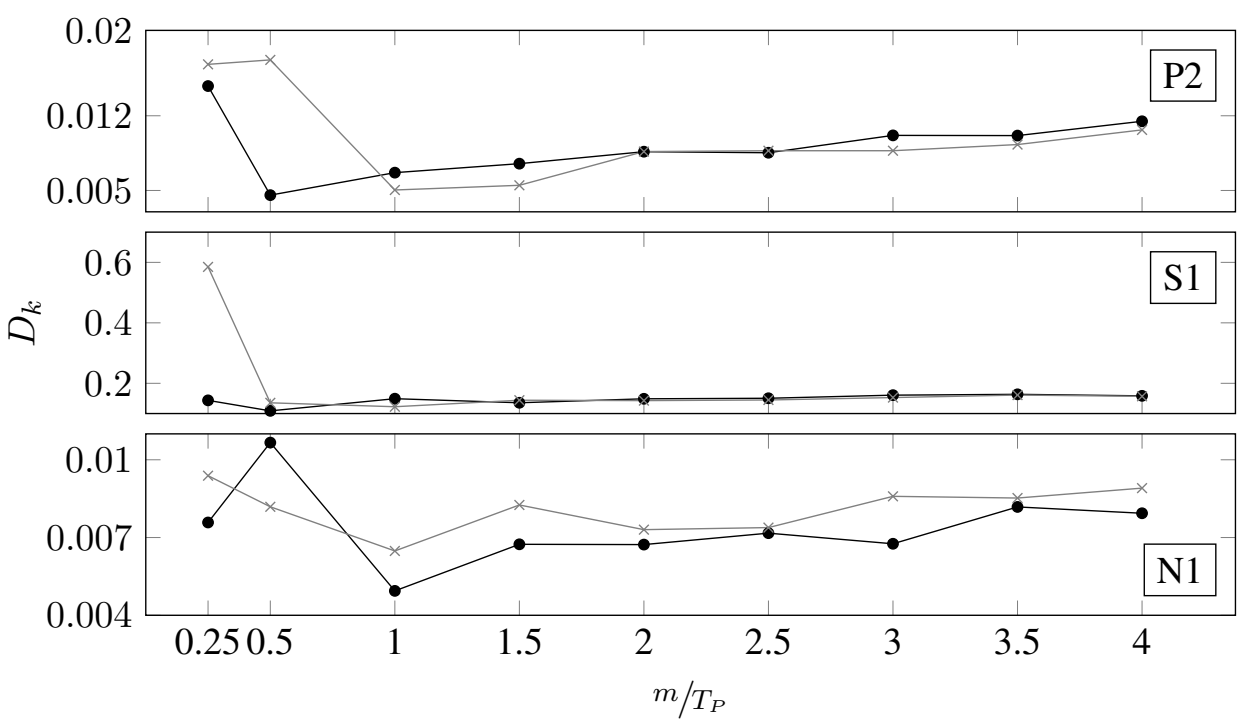

(a) Performance measure $D_{k}$.
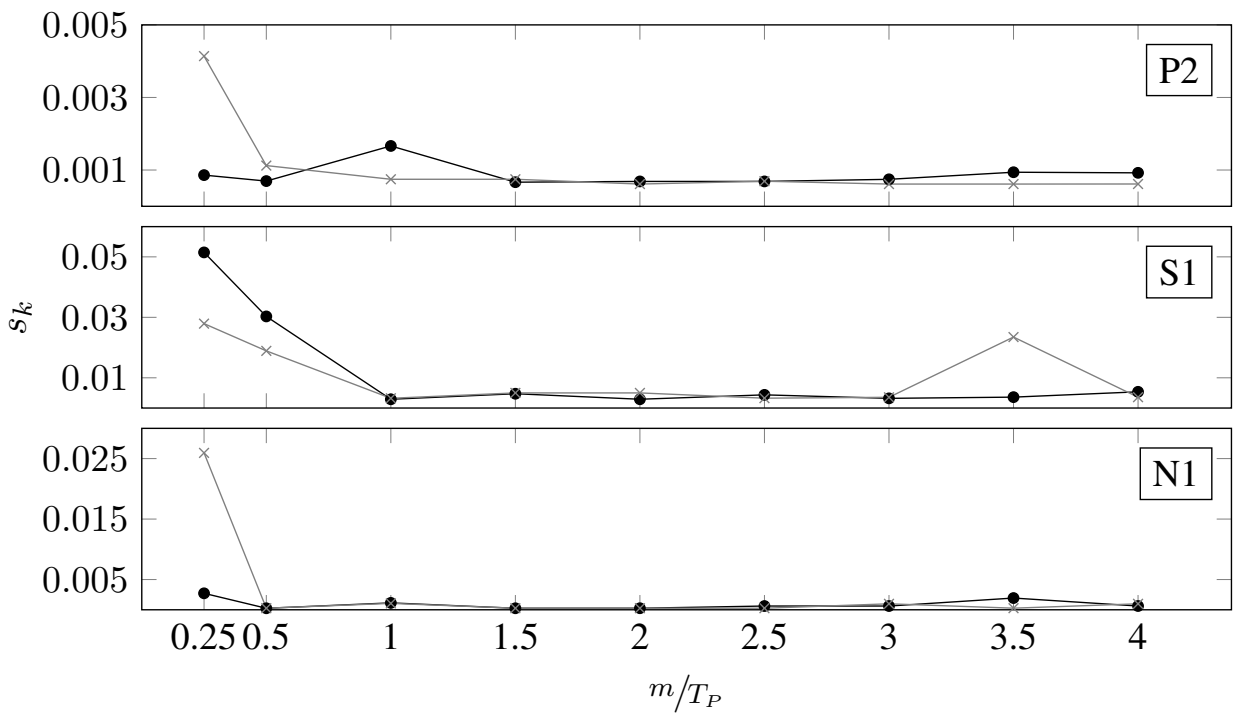

(b) Performance measure $s_{k}$.

Figure 13: Performance of the removal method as a function of $m / T_{P}$. Parameters fixed in the analyses were $\tau=1, \delta=1$ and $k=2$. Results are shown for each variable, and for the estimations based on the pre-transient reference (black line with round marker) and the post-transient reference (grey line with cross marker). 

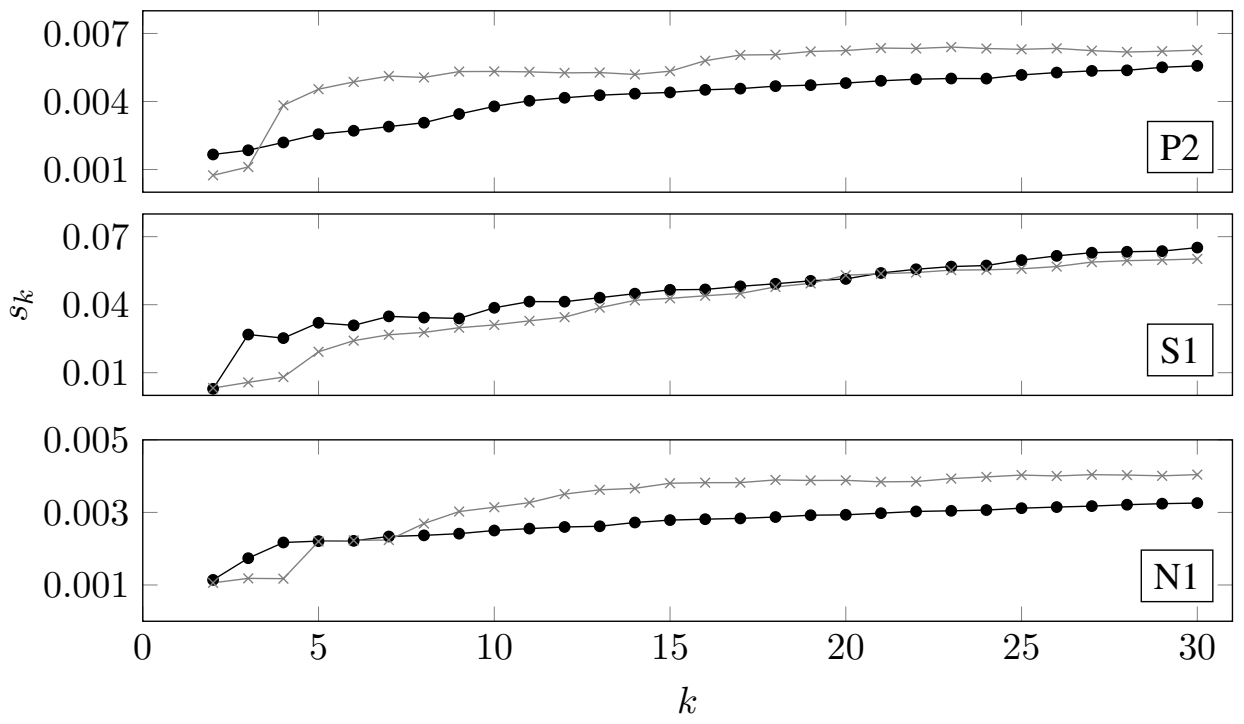

Figure 14: Performance measure $s_{k}$ as a function of $k$. Parameters fixed in the analyses were $\tau=1$, $\delta=1$ and $m=T_{P}=800$. Results are shown for each variable, and for the estimations based on the pre-transient reference (black line with round marker) and the post-transient reference (grey line with cross marker).

\subsubsection{Number of nearest neighbors $k$}

As explained above, the optimal value of parameter $k$ depends on the individual time series. Therefore, in a practical application a replacing segment should be computed for several $k$ values. For each $k$, the inconsistency index $s_{k}$ of the replacing segment is computed. The value of $k$ which minimizes $s_{k}$ is chosen for that application. The minimum of $s_{k}$ is expected at the first few values of $k$ because these correspond to segments adjacent to the nearest neighbors. The guideline is to attempt values of $k$ up to ten.

The inconsistency index $D_{k}$ is not used in the choice of $k$ because it will always increase with $k$. The reason is that $D_{k}$ derives from the distances between the reference embedded vector and its $k$ nearest neighbors (equation (11)).

Figure 14 demonstrates the use of index $s_{k}$ in the choice of $k$ for the development case study. Each plot shows the optimization results for each variable of the case study. Each plot combines the results based on the pre-transient reference (black line with round marker) and the post-transient reference (grey line with cross marker).

In this case study, $s_{k}$ increases monotonically with increasing $k$. In such a case, $k=2$ is recommended. Choosing $k=1$ is less recommended because the 
replacing segment $\left[\hat{x}_{t_{i}} \cdots \hat{x}_{t_{f}}\right]$ is more susceptible to any outliers that might be present in the single segment used.

The non-decreasing trend of $s_{k}$ in this case study can be explained by the strong sinusoidal dynamic of the system. Because uncertainty is small and outliers are rare in this system, none of the segments adjacent to the first few $k$ nearest neighbors is a bad predictor for the replacing segment $\left[\hat{x}_{t_{i}} \cdots \hat{x}_{t_{f}}\right]$. Because noise is common in a real system, the segments averaged into $\left[\begin{array}{lll}\hat{x}_{t_{i}} & \cdots & \hat{x}_{t_{f}}\end{array}\right]$ become increasingly different from each other, hence $s_{k}$ increases.

\subsubsection{Analysis workflow and summary of parameter values}

In a practical application of the removal method, the typical workflow would be:

1. Preparation of the data

2. Setting of parameters $m, \tau$, and $\delta$ based on $T_{P}$, the number of samples in one cycle of the oscillatory time series, as summarized in table 3 .

3. Computation of the steps of the algorithm described in sections 3.2.1 to 3.2.3.

4. Computation of a replacing segment for each value of $k$ from $k=1$ to $k=10$. For each value of $k$, the replacing segment is computed according to sections 3.2.4 and 3.2.5.

5. Computation of the error measure $s_{k}$ for each replacing segment, as exemplified in section 4.2.3.

6. Selection of the replacing segment with smallest $s_{k}$.

7. Reconstruction of the time series with the chosen replacing segment, as described in section 3.2.6.

Table 3 summarizes the guidelines for all the parameters, as well as their influence in the removal task. These guidelines are generic for any system. Parameters $m, \tau$, and $\delta$ are referred to $T_{P}$, the number of samples in one cycle of the oscillatory time series. The reason is that $T_{P}$ adapts the parameter value to the dynamics of each particular system.

\section{Application to industrial case study}

This section demonstrates the removal method on an industrial case study. The case study derives from routine operation of an industrial gas processing plant, and was provided courtesy of ABB Oil, Gas and Petrochemicals, Oslo, Norway. The top panel of Figure 15 shows measurements from a gas expansion-recompression section of the process. These include gas temperatures (T1, T2, and T3), and pressures (P1, and $\mathrm{P} 2)$, turbine speed (S1), and pressure on the bearings of the turbine 
Table 3: Summary of the roles and guidelines for the parameters. $T_{P}$ refers to the number of samples in one cycle of the oscillatory time series.

\begin{tabular}{|c|c|c|}
\hline \multicolumn{2}{|c|}{ ParametersInfluence } & Recommended value \\
\hline$\tau$ & $\begin{array}{l}\text { reduces accuracy of similarity measure } \\
\text { between the trends of embedded vectors, } \\
\text { and reduces computational time }\end{array}$ & $\begin{array}{l}\tau<T_{P} / 40 \\
\tau=1\end{array}$ \\
\hline$\delta$ & $\begin{array}{l}\text { omits possible embedded vectors from } \\
\text { the similarity assessment, and reduces } \\
\text { computational time }\end{array}$ & $\begin{array}{l}\delta<T_{P} / 40 \\
\delta=1\end{array}$ \\
\hline$m$ & $\begin{array}{l}\text { approximates the dimension of a state of } \\
\text { the system }\end{array}$ & $m=T_{P} / \tau$ \\
\hline$k$ & $\begin{array}{l}\text { defines the number of segments averaged } \\
\text { into the replacing segment }\end{array}$ & $\begin{array}{l}\text { Replacing segments } \\
\text { calculated for } \\
k=1: 10\end{array}$ \\
\hline
\end{tabular}



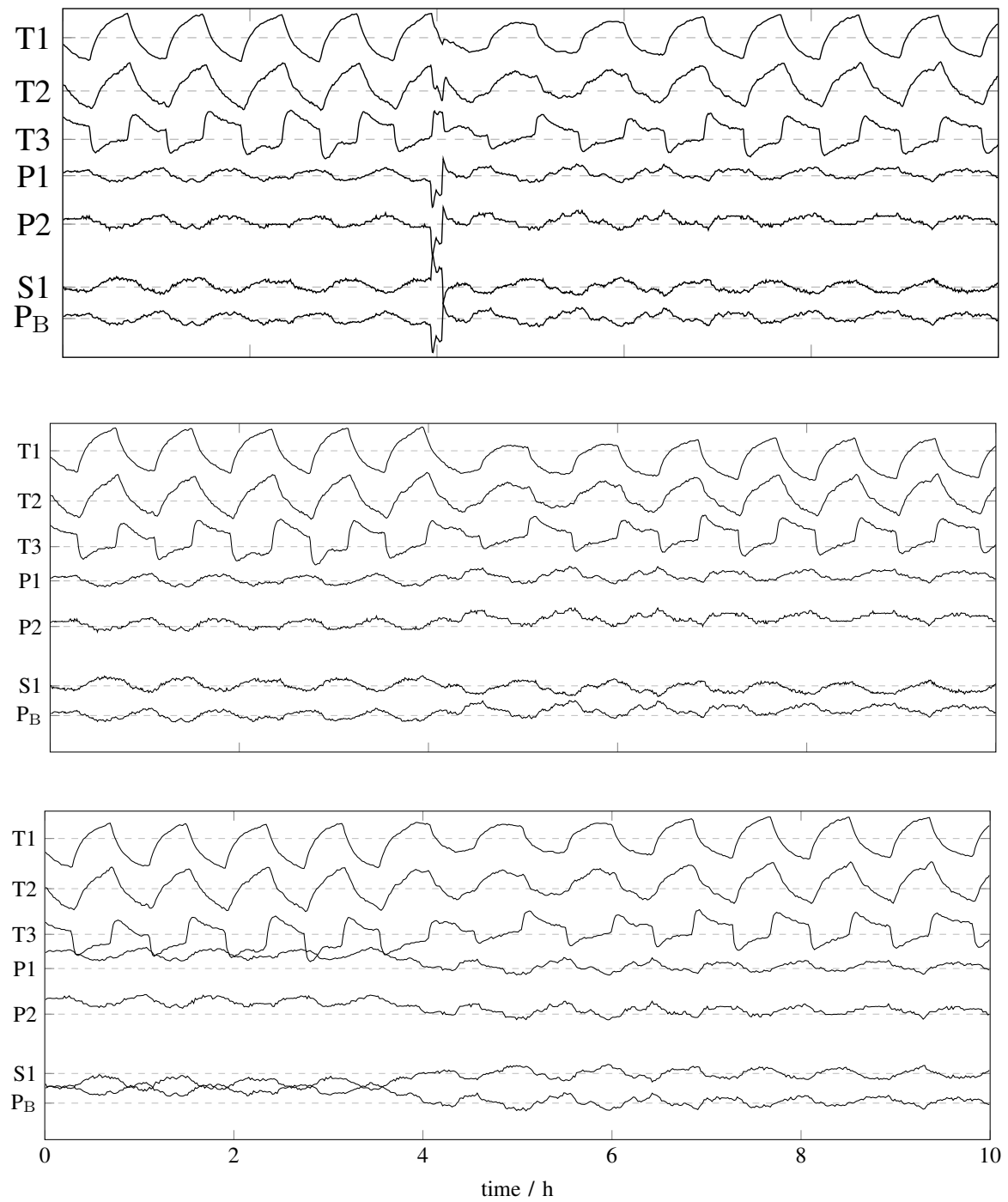

Figure 15: Original time series (top panel) and reconstructed time series based on the pre-transient reference (middle panel) and post-transient reference (bottom panel). 


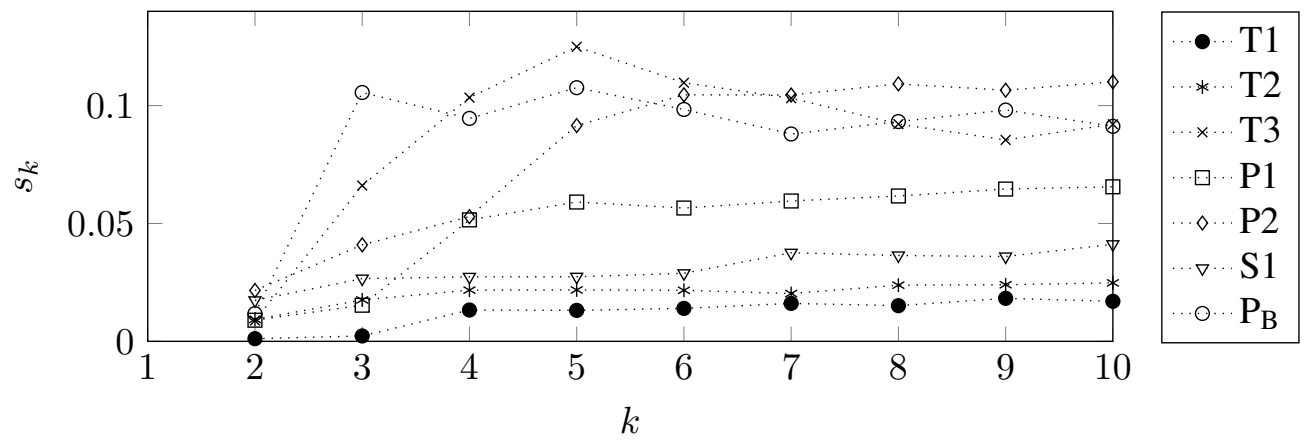

(a)

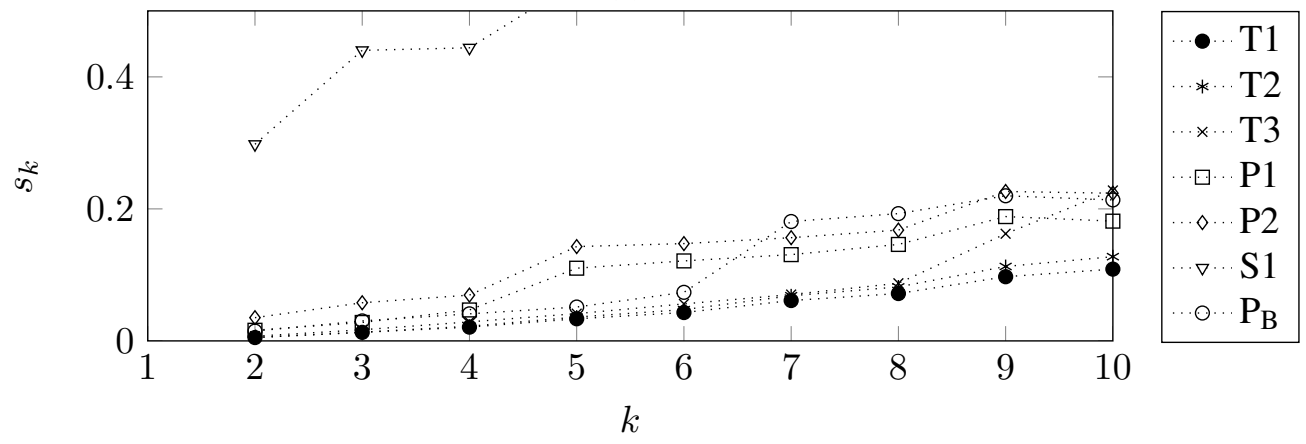

(b)

Figure 16: Influence of $k$ on the inconsistency index $s_{k}$ for the pre-transient references (a) and posttransient references (b).

$\left(\mathrm{P}_{\mathrm{B}}\right)$. The transient affecting these measurements occurs around hour 4 in the figure. Each time series has 1200 samples taken with a sampling interval of $30 \mathrm{~s}$. The expectation for this test case study is that the nearest neighbors imputation method correctly removes the transients. The reconstructed time series should be an estimate of what the measurement would have been had the transient not been present.

Following the recommendations in section 4, parameters $\tau$ and $\delta$ are set to one. Parameter $m$ is set to 100 , which is approximately the number of samples in one cycle of the oscillations. This length is the same in all measurements.

Parameter $k$ is varied between two and ten. The resulting inconsistency indices $s_{k}$ for all the measurements are plotted in Figure 16a for the pre-transient references and in Figure 16b for the post-transient references. In all cases, $s_{k}$ is minimum at $k=2$, hence this value is used to obtain the reconstructed time series. 
Table 4: Inconsistency indices $D_{k}$ and $s_{k}$ for the new times series of the measurement in the test case study.

\begin{tabular}{lccccc}
\hline \multirow{2}{*}{ Measurement } & \multicolumn{2}{c}{$D_{k}$} & & \multicolumn{2}{c}{$s_{k}$} \\
\cline { 2 - 3 } \cline { 5 - 6 } & Previous & Subsequent & & Previous & Subsequent \\
\hline $\mathrm{T} 1$ & $\mathbf{0 . 0 0 7}$ & 0.018 & & $\mathbf{0 . 0 0 1}$ & 0.005 \\
$\mathrm{~T} 2$ & $\mathbf{0 . 0 0 9}$ & 0.039 & & 0.009 & $\mathbf{0 . 0 0 5}$ \\
$\mathrm{T} 3$ & $\mathbf{0 . 0 4 1}$ & 0.271 & & 0.009 & $\mathbf{0 . 0 0 7}$ \\
$\mathrm{P} 1$ & $\mathbf{0 . 0 6 5}$ & 0.215 & & $\mathbf{0 . 0 0 9}$ & 0.016 \\
$\mathrm{P} 2$ & $\mathbf{0 . 0 9 5}$ & 0.226 & & $\mathbf{0 . 0 2 2}$ & 0.035 \\
$\mathrm{~S} 1$ & $\mathbf{0 . 0 7 1}$ & 0.192 & & $\mathbf{0 . 0 1 7}$ & 0.298 \\
$\mathrm{P}_{\mathrm{B}}$ & $\mathbf{0 . 0 9 4}$ & 0.229 & & $\mathbf{0 . 0 1 2}$ & 0.015 \\
\hline
\end{tabular}

The middle and bottom panels of Figure 15 show the reconstructed time series, which derive from the pre- and post-transient references, respectively. The comparison shows that the removal method and the parameters proposed are able to generate time series which are free of transients and maintain the original oscillatory dynamics.

The reconstructed time series are different for the results based on the pre- and post-transient references. Visual analysis suggests that the time series based on pretransient references present less distortion of the original time series. This is more evident for measurements $\mathrm{P} 1, \mathrm{P} 2, \mathrm{~S} 1$ and $\mathrm{P}_{\mathrm{B}}$. These observations are confirmed by the inconsistency indices $D_{k}$ and $s_{k}$, which are indicated in table 4 .

It is generally not possible to know in advance which reference embedded vectors will lead to the most adequate reconstructed time series. In the industrial case study, the two oscillation cycles after the transient disturbance are different from the other cycles in the measurement, as shown in Figure 15. Therefore, the replacing segment will join two sections of the measurement which have different trends. In this case study, the joining is smoother with the replacing segment based on the pre-transient reference.

The conclusions from these observations are that it is important to calculate reconstructed time series based on both pre- and post-transient references, and to have indices such as $D_{k}$ and $s_{k}$ which can decide on the best reconstructed time series on a case-to-case basis. 


\section{Conclusions}

This paper has presented a method to remove transient disturbances from otherwise oscillating time series. Such a method is needed because transient disturbances affect the reliability of several oscillation analysis methods.

The method is based on a nearest neighbors imputation technique. This technique works based on the assumption that the system generating the time series is, to some extent, deterministic. The system can be linear or nonlinear. Time series with repeating patterns may be generated by nonlinear deterministic systems. The idea is to consider a segment of the time series adjacent to the removed transient disturbance, and look for its $k$ most similar segments in the time series. These are known as the $k$ nearest neighbors. The segments adjacent to the $k$ nearest neighbors are then used as replacements for the removed transient disturbance.

The paper also presented two indices to assess inconsistencies associated with the replacing segment. The purpose of the indices is to measure the agreement of the replacing segment to the underlying dynamics of the original time series. The inconsistency indices were used in analysing the sensitivity of the removal method to its parameters and in optimizing the values of those parameters.

The results showed that the method and parameters are able to generate time series which are free of transients and agree with the original oscillatory dynamics. The method works in any measurement that has some repeating patterns, even if not purely periodic.

The inconsistency indices are an additional merit of the transients removal method because they agree with visual analyses of the adequacy of the reconstructed time series. This agreement to the visual evaluation means that the inconsistency indices enable the automation of the method.

The limitations of the method are the need for measurements with regular sampling rate, also known as equidistant sampling, and the possibility of distortion relative to the original time series in the case that the original time series has different trends before and after the transient.

\section{Acknowledgment}

The authors gratefully acknowledge the financial support from the Portuguese Foundation for Science and Technology (FCT) under Fellowship SFRH/BD/61384/2009 and the Marie Curie FP7-IAPP project "REAL-SMART - Using real-time measurements for monitoring and management of power transmission dynamics for the Smart Grid", Contract No: PIAP-GA-2009-251304.

The authors would also like to thank P. Lipnicki, D. Lewandowski, and M. Wojcik of ABB Corporate Research Poland for enabling and helping with the experimental 
work, and K. Rapp of ABB A.S. for providing data and process insights to support this paper.

\section{References}

[1] N. F. Thornhill, A. Horch, Advances and new directions in plant-wide disturbance detection and diagnosis, Control Engineering Practice 15 (10) (2007) 1196-1206.

[2] Y. A. W. Shardt, Y. Zhao, K. H. Lee, X. Yu, B. Huang, S. L. Shah, Determining the state of a process control system: Current trends and future challenges, The Canadian Journal of Chemical Engineering 90 (2) (2012) 217-245.

[3] M. A. A. S. Choudhury, S. L. Shah, N. F. Thornhill, Diagnosis of poor control-loop performance using higher-order statistics, Automatica 40 (10) (2004) 1719-1728.

[4] N. F. Thornhill, Finding the source of nonlinearity in a process with plantwide oscillation, IEEE Transactions on Control Systems Technology 13 (3) (2005) 434-443.

[5] S. J. Qin, Survey on data-driven industrial process monitoring and diagnosis, Annual Reviews in Control 36 (2) (2012) 220 - 234.

[6] V. Venkatasubramanian, R. Rengaswamy, K. Yin, S. N. Kavuri, A review of process fault detection and diagnosis: Part I: quantitative model-based methods, Computers \& Chemical Engineering 27 (3) (2003a) 293-311.

[7] V. Venkatasubramanian, R. Rengaswamy, S. N. Kavuri, A review of process fault detection and diagnosis: Part II: qualitative models and search strategies, Computers \& Chemical Engineering 27 (3) (2003b) 313-326.

[8] V. Venkatasubramanian, R. Rengaswamy, S. N. Kavuri, K. Yin, A review of process fault detection and diagnosis: Part III: process history based methods, Computers \& Chemical Engineering 27 (3) (2003c) 327-346.

[9] M. Jelali, C. Scali, Detection and Diagnosis of Stiction in Control Loops: State of the Art and Advanced Methods, 2010, Ch. Comparative Study of Valve-stiction-detection Methods, pp. 295-358.

[10] I. M. Cecílio, J. R. Ottewill, J. Pretlove, N. F. Thornhill, Nearest neighbors method for detecting transient disturbances in process and electromechanical systems., Journal of Process Control 24 (9) (2014) 1382-1393. 
[11] J. Canny, A computational approach to edge detection, Pattern Analysis and Machine Intelligence, IEEE Transactions on (6) (1986) 679-698.

[12] M. R. Maurya, R. Rengaswamy, V. Venkatasubramanian, Fault diagnosis using dynamic trend analysis: A review and recent developments, Engineering Applications of Artificial Intelligence 20 (2) (2007) 133-146.

[13] D. C. Montgomery, Introduction to Statistical Quality Control, John Wiley \& Sons Inc., New York, 2013.

[14] M. Misra, H. H. Yue, S. J. Qin, C. Ling, Multivariate process monitoring and fault diagnosis by multi-scale PCA, Computers \& Chemical Engineering 26 (9) (2002) 1281-1293.

[15] I. M. Cecílio, J. R. Ottewill, H. Fretheim, N. F. Thornhill, Multivariate detection of transient disturbances for uni- and multi-rate systems, IEEE Transactions on Control System Technology 23 (4) (2015) 1477-1493.

[16] R. Dunia, S. J. Qin, Subspace approach to multidimensional fault identification and reconstruction, AIChE Journal 44 (8) (1998) 1813-1831.

[17] H. H. Yue, S. J. Qin, Reconstruction-based fault identification using a combined index, Industrial \& Engineering Chemistry Research 40 (20) (2001) 4403-4414.

[18] S. J. Qin, Statistical process monitoring: basics and beyond, Journal of Chemometrics 17 (8-9) (2003) 480-502.

[19] E. L. Russell, L. H. Chiang, R. D. Braatz, Data-driven Methods for Fault Detection and Diagnosis in Chemical Processes, $1^{\text {st }}$ Edition, Springer, 2000.

[20] I. W. Selesnick, Resonance-based signal decomposition: A new sparsityenabled signal analysis method, Signal Processing 91 (12) (2011) 2793-2809.

[21] L. M. Briceno-Arias, P. L. Combettes, J.-C. Pesquet, N. Pustelnik, Proximal algorithms for multicomponent image recovery problems, Journal of Mathematical Imaging and Vision 41 (1-2) (2011) 3-22.

[22] H. Kantz, T. Schreiber, Nonlinear Time Series Analysis, Cambridge University Press, 2003.

[23] M. B. Kennel, S. Isabelle, Method to distinguish possible chaos from colored noise and to determine embedding parameters, Physical Review A 46 (6) (1992) 3111. 
[24] J. Chen, J. Shao, Nearest neighbor imputation for survey data, Journal of Official Statistics 16 (2) (2000) 113-132.

[25] M. Bauer, J. W. Cox, M. H. Caveness, J. J. Downs, N. F. Thornhill, Nearest neighbors methods for root cause analysis of plantwide disturbances, Industrial \& Engineering Chemistry Research 46 (18) (2007) 5977-5984.

[26] M. Stockmann, R. Haber, U. Schmitz, Source identification of plant-wide faults based on $k$ nearest neighbor time delay estimation, Journal of Process Control 22 (3) (2012) 583-598. 